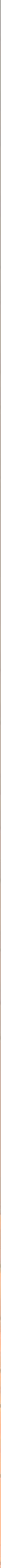




\title{
The existential theory of equicharacteristic henselian valued fields
}

\author{
Sylvy Anscombe and Arno Fehm
}

\begin{abstract}
We study the existential (and parts of the universal-existential) theory of equicharacteristic henselian valued fields. We prove, among other things, an existential Ax-Kochen-Ershov principle, which roughly says that the existential theory of an equicharacteristic henselian valued field (of arbitrary characteristic) is determined by the existential theory of the residue field; in particular, it is independent of the value group. As an immediate corollary, we get an unconditional proof of the decidability of the existential theory of $\mathbb{F}_{q}((t))$.
\end{abstract}

\section{Introduction}

We study the first order theory of a henselian valued field $(K, v)$ in the language of valued fields. For residue characteristic zero, this theory is well-understood through the celebrated Ax-Kochen-Ershov (AKE) principles, which state that, in this case, the theory of $(K, v)$ is completely determined by the theory of the residue field $K v$ and the theory of the value group $v K$ (see, e.g., [Prestel and Delzell 2011, §4.6]). In other words, if a sentence holds in one such valued field, then it holds in any other with elementarily equivalent residue field and value group (the transfer principle). As a consequence, one gets that the theory of $(K, v)$ is decidable if and only if the theory of the residue field and the theory of the value group are decidable.

Some of this theory can be carried over to certain mixed characteristic henselian valued fields such as the fields of $p$-adic numbers $\mathbb{Q}_{p}$, whose theory was axiomatised and proven to be decidable by Ax-Kochen and Ershov in 1965. However, for henselian valued fields of positive characteristic, no such general principles are available. For example, in [Kuhlmann 2001], it is shown that the theory of characteristic $p>0$ henselian valued fields with value group elementarily equivalent to $\mathbb{Z}$ and residue field $\mathbb{F}_{p}$ is incomplete. It is not known whether there is a suitable modification of the AKE principles that hold for arbitrary henselian valued fields

During this research, Anscombe was funded by EPSRC grant EP/K020692/1.

MSC2010: primary 03C60; secondary 12L12, 12J10, 11U05, 12L05.

Keywords: model theory, henselian valued fields, decidability, diophantine equations. 
of positive characteristic, and the decidability of the field of formal power series $\mathbb{F}_{q}((t))$ is a long-standing open problem.

For the first problem, the most useful approximations are AKE principles for certain classes of valued fields, most notably F.-V. Kuhlmann's recently published work [2014] on the model theory of tame fields. For the second problem, the best known result is by Denef and Schoutens [2003], who proved that resolution of singularities in positive characteristic would imply that the existential theory of $\mathbb{F}_{q}((t))$ is decidable (i.e., Hilbert's tenth problem for $\mathbb{F}_{q}((t))$ has a positive solution).

In this work, we take a different approach at deepening our understanding of the positive characteristic case: instead of limiting ourselves to certain classes of valued fields, we attempt to prove results for arbitrary equicharacteristic henselian valued fields, but (having results like Denef-Schoutens in mind) instead restrict to existential or slightly more general sentences. The technical heart of this work is a study of transfer principles for certain universal-existential sentences, which builds on the aforementioned [Kuhlmann 2014]; see the results in Section 5. While some of these general results will have applications for example in the theory of definable valuations (see [Anscombe and Koenigsmann 2014; Cluckers et al. 2013; Fehm 2015; Prestel 2015] for some of the recent developments), in this work we then restrict this machinery to existential sentences and deduce the following result (cf. Theorem 6.5):

Theorem 1.1. For any field $F$, the theory $T$ of equicharacteristic henselian nontrivially valued fields with residue field which models both the existential and universal theories of $F$ is $\exists$-complete, i.e., for any existential sentence $\phi$ either $T \models \phi$ or $T \models \neg \phi$.

Note that the value group plays no role here: the existential theory of an equicharacteristic henselian nontrivially valued field is determined solely by its residue field. From this theorem, we obtain an AKE principle for $\exists$-sentences (cf. Corollary 7.2):

Corollary 1.2. Let $(K, v),(L, w)$ be equicharacteristic henselian nontrivially valued fields. If the residue fields $K v$ and $L w$ have the same existential theory, then so do the valued fields $(K, v)$ and $(L, w)$.

Moreover, we conclude the following corollary on decidability (cf. Corollary 7.5):

Corollary 1.3. Let $(K, v)$ be an equicharacteristic henselian valued field. The following are equivalent:

(1) The existential theory of $K v$ in the language of rings is decidable.

(2) The existential theory of $(K, v)$ in the language of valued fields is decidable.

As an immediate consequence, we get the first unconditional proof of the decidability of the existential theory of $\mathbb{F}_{q}((t))$ (cf. Corollary 7.7). Note, however, 
that the conditional result in [Denef and Schoutens 2003] is for a language with a constant for $t$ - Section 7 also contains a brief discussion of this difference.

As indicated above, these results are essentially known in residue characteristic zero (cf. Remark 7.3), but are new in positive characteristic. However, each of the above results fails if "equicharacteristic" is dropped or replaced by "mixed characteristic", in contrast to the mixed characteristic AKE principles mentioned above (cf. Remark 7.4 and Remark 7.6).

\section{Valued fields}

For a valued field $(K, v)$ we denote by $v K=v\left(K^{\times}\right)$its value group, by $\mathcal{O}_{v}$ its valuation ring, and by $K v=\left\{a v \mid a \in \mathcal{O}_{v}\right\}$ its residue field. For standard definitions and facts about henselian valued fields we refer the reader to [Engler and Prestel 2005]. As a rule, if $L / K$ is a field extension to which the valuation $v$ can be extended uniquely, we denote also this unique extension by $v$. This applies in particular if $v$ is henselian, and for the perfect hull $L=K^{\text {perf }}$ of $K$. We will make use of the following well-known fact:

Lemma 2.1. Let $(K, v)$ be a valued field and let $F / K v$ be any field extension. Then there is an extension of valued fields $(L, w) /(K, v)$ such that $L w / K v$ is isomorphic to the extension $F / K v$.

Proof. See, e.g., [Kuhlmann 2004, Theorem 2.14].

The next lemma is also probably well known, but for lack of reference we sketch a proof, which closely follows [Kuhlmann 2011, Lemma 9.30].

Definition 2.2. Let $(K, v)$ be a valued field. A partial section (of the residue homomorphism) is a map $f: E \rightarrow K$, for some subfield $E \subseteq K v$, which is an

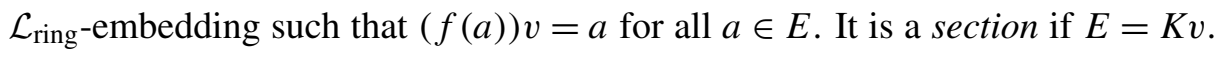

Lemma 2.3. Let $(K, v)$ be an equicharacteristic henselian valued field, let $E \subseteq K v$ be a subfield of the residue field, and suppose that there is a partial section $f: E \rightarrow K$. If $F / E$ is a separably generated subextension of $K v / E$ then we may extend $f$ to a partial section $F \rightarrow K$.

Proof. Write $L_{1}:=f(E)$. Let $T$ be a separating transcendence base for $F / E$ and, for each $t \in T$, choose $s_{t} \in K$ such that $s_{t} v=t$. Then $S:=\left\{s_{t} \mid t \in T\right\}$ is algebraically independent over $L_{1}$. Thus we may extend $f$ to a partial section $E(T) \rightarrow L_{1}(S)$ by sending $t \mapsto s_{t}$.

Let $L_{2}$ be the relative separable algebraic closure of $L_{1}(S)$ in $K$. By Hensel's lemma, $L_{2} v$ is separably algebraically closed in $K v$. Thus $F$ is contained in $L_{2} v$. Since $v$ is trivial on $L_{2}$, the restriction of the residue map to $L_{2}$ is an isomorphism $L_{2} \rightarrow L_{2} v$. Thus the restriction to $F$ of the inverse of the residue map is a partial section $F \rightarrow K$ which extends $f$, as required. 
Recall that a valued field $(K, v)$ of residue characteristic $p$ is tame if it is henselian, the value group $v K$ is $p$-divisible, the residue field $K v$ is perfect, and $(K, v)$ is defectless, i.e., for every finite extension $L / K$,

$$
[L: K]=[L v: K v] \cdot[v L: v K] .
$$

Proposition 2.4. Let $(K, v)$ be a valued field. There exists an extension $\left(K^{t}, v^{t}\right)$ of $(K, v)$ such that $\left(K^{t}, v^{t}\right)$ is tame, $K^{t}$ is perfect, $v^{t} K^{t}=\frac{1}{p^{\infty}} v K$, and $K^{t} v^{t}=K v^{\text {perf }}$. Proof. In the special case $\operatorname{char}(K)=\operatorname{char}(K v)$, any maximal immediate extension of $K^{\text {perf }}$ satisfies the claim. In general, [Kuhlmann et al. 1986, Theorem 2.1, Proposition 4.1, and Proposition 4.5(i)] gives such a $K^{t}$ that is in addition algebraic over $K$.

\section{Model theory of valued fields}

Let

$$
\mathcal{L}_{\text {ring }}=\{+,-, \cdot, 0,1\}
$$

be the language of rings and let

$$
\mathcal{L}_{\mathrm{vf}}=\left\{+^{K},-^{K},{ }^{K}, 0^{K}, 1^{K},+^{\Gamma},<^{\Gamma}, 0^{\Gamma}, \infty^{\Gamma},+^{k},-{ }^{k}, \cdot{ }^{k}, 0^{k}, 1^{k}, v, \text { res }\right\}
$$

be a three sorted language for valued fields (like the Denef-Pas language, but without an angular component) with a sort $K$ for the field itself, a sort $\Gamma \cup\{\infty\}$ for the value group with infinity, and a sort $k$ for the residue field, as well as both the valuation map $v$ and the residue map res, which we interpret as the constant $0^{k}$ map outside the valuation ring. For a field $C$, we let $\mathcal{L}_{\text {ring }}(C)$ and $\mathcal{L}_{\mathrm{vf}}(C)$ be the languages obtained by adding symbols for elements of $C$. In the case of $\mathcal{L}_{\mathrm{vf}}(C)$, the constant symbols are added to the field sort $K$.

A valued field $(K, v)$ gives rise in the usual way to an $\mathcal{L}_{\mathrm{vf}}$-structure

$$
(K, v K \cup\{\infty\}, K v, v, \text { res }),
$$

where $v K$ is the value group, $K v$ is the residue field, and res is the residue map. For notational simplicity, we will usually write $(K, v)$ to refer to the $\mathcal{L}_{\mathrm{vf}}$-structure it induces. For further notational simplicity, we write $(K, D)$ instead of $\left(K,\left(d_{c}\right)_{c \in C}\right)$, where $D=\left\{d_{c} \mid c \in C\right\}$ is the set of interpretations of the constant symbols. Combining these two simplifications, we write $(K, v, D)$ for the $\mathcal{L}_{\text {vf }}(C)$-structure

$$
\left(K, v K \cup\{\infty\}, K v, v \text {, res, }\left(d_{c}\right)_{c \in C}\right) .
$$

We also write $D v$ for the set of residues of elements from $D$.

As usual, we say that an $\mathcal{L}_{\text {vf }}(C)$-formula is an $\exists$-formula if it is logically equivalent to a formula in prenex normal form with only existential quantifiers (over any of the three sorts). We say that an $\mathcal{L}_{\mathrm{vf}}(C)$-sentence is an $\forall^{k} \exists$-sentence if it is 
logically equivalent to a sentence of the form $\forall \boldsymbol{x} \psi(\boldsymbol{x})$, where $\psi$ is an $\exists$-formula and the universal quantifiers range over the residue field sort.

Let $(K, v, D) \subseteq(L, w, E)$ be an extension of $\mathcal{L}_{\mathrm{vf}}(C)$-structures. Note that $d_{c}=e_{c}$ for all $c \in C$. We say that certain $\mathcal{L}_{\mathrm{vf}}(C)$-sentences $\phi$ go up from $K$ to $L$ if $(K, v, D) \models \phi$ implies that $(L, w, E) \models \phi$. For examples, $\exists$-sentences always go up every extension. Furthermore, if $(L, w) /(K, v)$ is an extension of valued fields such that $L w / K v$ is trivial, then $\forall^{k} \exists-\mathcal{L}_{\mathrm{vf}}(K)$-sentences go up from $(K, v)$ to $(L, w)$. Although the previous statement is not referenced directly, it underlies many of the arguments in Section 5.

Lemma 3.1. Let $L / K$ be an extension of fields. If $K \preceq_{\exists} L$, then $K^{\text {perf }} \preceq_{\exists} L^{\text {perf }}$.

Proof. This is clear, since $K^{\text {perf }}=\bigcup_{n} K^{p^{-n}}$ and $L^{\text {perf }}=\bigcup_{n} L^{p^{-n}}$, and the Frobenius gives that $K^{p^{-n}} \preceq_{\exists} L^{p^{-n}}$ for all $n$.

F.-V. Kuhlmann [2014] proves the following on the model theory of tame fields:

Proposition 3.2. The elementary class of tame fields has the relative embedding property. That is, for tame fields $(K, v)$ and $(L, w)$ with common subfield $(F, u)$, if

(1) $(F, u)$ is defectless,

(2) $(L, w)$ is $|K|^{+}$-saturated,

(3) $v K / u F$ is torsion-free and $K v / F u$ is separable, and

(4) there are embeddings $\rho: v K \rightarrow w L$ (over $u F)$ and $\sigma: K v \rightarrow L w($ over $F u)$, then there exists an embedding $\iota:(K, v) \rightarrow(L, w)$ over $(F, u)$ which respects $\rho$ and $\sigma$.

Proof. See [Kuhlmann 2014, Theorem 7.1]. (Note that this result is stated in the language

$$
\mathcal{L}_{\mathrm{vf}}^{\prime}=\left\{+,-, \cdot{ }^{-1}, 0,1, O\right\},
$$

where $O$ is a binary predicate which is interpreted in a valued field $(K, v)$ so that $O(a, b)$ if and only if $v a \geq v b$. However, the exact choice of language does not directly affect us.)

From Proposition 3.2, Kuhlmann deduces the following AKE principle:

Theorem 3.3. The class of tame fields is an $\mathrm{AKE}^{\preceq}$-class: if $(L, w) /(K, v)$ is an extension of tame fields with $v K \preceq w L$ and $K v \preceq L w$, then $(K, v) \preceq(L, w)$.

Proof. See [Kuhlmann 2014, Theorem 1.4]. 


\section{Power series fields}

For a field $F$ and an ordered abelian group $\Gamma$ we denote by $F((\Gamma))$ the field of generalised power series with coefficients in $F$ and exponents in $\Gamma$; see, e.g., [Efrat $2006, \S 4.2]$. We identify $F((\mathbb{Z}))$ with the field of formal power series $F((t))$ and denote the power series valuation on any subfield of any $F((\Gamma))$ by $v_{t}$.

Lemma 4.1. A field $\left(F((\Gamma)), v_{t}\right)$ of generalised power series is maximal. In particular, it is tame if and only if $F$ is perfect and $\Gamma$ is $p$-divisible.

Proof. See [Efrat 2006, Theorem 18.4.1] and note that maximal implies henselian and defectless.

Proposition 4.2. Let $A$ be a complete discrete (i.e., with value group $\mathbb{Z}$ ) equicharacteristic valuation ring. Let $F \subseteq A$ be a set of representatives for the residue classes which forms a field. Let $s \in A$ be a uniformiser (i.e., an element of least positive value). Then $A$ is isomorphic to $F \llbracket s \rrbracket$ by an isomorphism which fixes $F$ pointwise. Proof. See [Serre 1979], Chapter 2 Proposition 5 and the discussion following the example.

Corollary 4.3. Let $F$ be a field and let $E / F((t))$ be a finite extension such that $E v_{t}=F$. Then $\left(E, v_{t}, F\right)$ is isomorphic to $\left(F((s)), v_{s}, F\right)$. This applies in particular to finite extensions of $F((t))$ inside $F((\mathbb{Q}))$.

Proof. We are already provided with a section since $F \subseteq F((t)) \subseteq E$ and $E v_{t}=F$. Since $E / F((t))$ is finite, $E$ is also a complete discrete equicharacteristic valued field (cf. [Serre 1979, Chapter 2 Proposition 3]). By Proposition 4.2, there is an $F$-isomorphism of valued fields $E \rightarrow F((s))$.

Definition 4.4. We denote by $F(t)^{h}$ the henselization of $F(t)$ with respect to $v_{t}$, i.e., the relative algebraic closure of $F(t)$ in $F((t))$, and by $F((t))^{\mathbb{Q}}$ the relative algebraic closure of $F((t))$ in $F((\mathbb{Q}))$.

Lemma 4.5. For any field $F$ we have $\left(F(t)^{h}, v_{t}\right) \preceq_{\exists}\left(F((t)), v_{t}\right)$.

Proof. See [Kuhlmann 2014, Theorem 5.12].

The following proposition may be deduced from the more general [Kuhlmann 2014, Lemma 3.7], but we give a proof in this special case for the convenience of the reader.

Proposition 4.6. If $F$ is perfect, then $F((t))^{\mathbb{Q}}$ is tame.

Proof. We have that $F((t))^{\mathbb{Q}} v_{t}=F$ is perfect and $v_{t} F((t))^{\mathbb{Q}}=\mathbb{Q}$ is $p$-divisible. Moreover, as an algebraic extension of the henselian field $F((t)), F((t))^{\mathbb{Q}}$ is henselian. It remains to show that $F((t))^{\mathbb{Q}}$ is defectless.

Let $E / F((t))^{\mathbb{Q}}$ be a finite extension of degree $n$. Since $F((\mathbb{Q}))$ is perfect, so is $F((t))^{\mathbb{Q}}$, and hence $F((\mathbb{Q})) / F((t))^{\mathbb{Q}}$ is regular. Therefore, if $E^{\prime}=F((\mathbb{Q})) \cdot E$ 
denotes the compositum of $F((\mathbb{Q}))$ and $E$ in an algebraic closure of $F((\mathbb{Q}))$, then $\left[E^{\prime}: F((\mathbb{Q}))\right]=n$. Since $F((\mathbb{Q}))$ is maximal (Lemma 4.1), $E^{\prime} / F((\mathbb{Q}))$ is defectless. So since $\left(F((\mathbb{Q})), v_{t}\right)$ is henselian and $v_{t} F((\mathbb{Q}))=\mathbb{Q}$ is divisible, we get that $\left[E^{\prime} v_{t}: F\right]=n$. Since $E^{\prime} v_{t} / F$ is separable, we can assume without loss of generality that $F^{\prime}:=E^{\prime} v_{t} \subseteq E^{\prime}$ (Lemma 2.3).

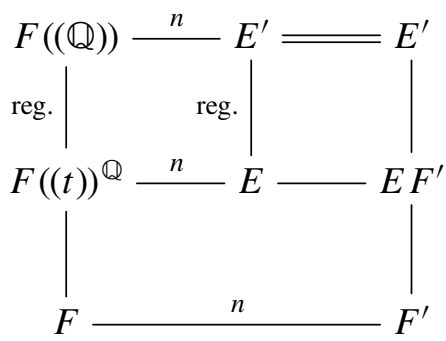

The extension $E^{\prime} / E$ is also regular, since $E / F((t))^{\mathbb{Q}}$ is algebraic. In particular, $E$ is relatively algebraically closed in $E^{\prime}$; so since $E F^{\prime} / E$ is algebraic we have that $F^{\prime} \subseteq E$. Thus $E v_{t}=F^{\prime}$, which shows that $E / F((t))^{\mathbb{Q}}$ is defectless.

In particular, Theorem 3.3 implies that $F((t))^{\mathbb{Q}} \preceq F((\mathbb{Q}))$. We therefore get the following picture:

$$
F(t) \stackrel{\text { alg. }}{=} F(t)^{h} \stackrel{\unlhd_{\exists}}{=} F((t)) \stackrel{\text { alg. }}{=} F((t))^{\mathbb{Q}} \stackrel{\preceq}{=} F((\mathbb{Q}))
$$

\section{The transfer of universal-existential sentences}

Throughout this section $F / C$ will be a separable extension of fields of characteristic $p$. We show that the truth of $\forall^{k} \exists$-sentences transfers between various valued fields. Usually the valued fields considered will have only elementarily equivalent residue fields. However, for convenience, we will sometimes discuss $\exists$-sentences with additional parameters from the residue field.

Lemma 5.1 (going down from $\boldsymbol{F}((\Gamma))$ ). Suppose that $F$ is perfect. Let $\phi$ be an $\exists$ - $\mathcal{L}_{\mathrm{vf}}(F)$-sentence, let $F \preceq \boldsymbol{F}$ be an elementary extension, and let $\Gamma$ be an ordered abelian group. If $\left(\boldsymbol{F}((\Gamma)), v_{t}, F\right) \models \phi$, then $\left(F(t)^{h}, v_{t}, F\right) \models \phi$.

Proof. Without loss of generality we may assume that $\Gamma$ is nontrivial. For notational simplicity, we suppress the parameters $F$ from the notation. Let $\Delta$ be the divisible hull of $\Gamma$. Then $\left(\boldsymbol{F}((\Gamma)), v_{t}\right) \subseteq\left(\boldsymbol{F}((\Delta)), v_{t}\right)$, and existential sentences "go up", so $\left(\boldsymbol{F}((\Delta)), v_{t}\right) \models \phi$.

Choose an embedding of $\mathbb{Q}$ into $\Delta$; this induces an embedding $\left(F((\mathbb{Q})), v_{t}\right) \subseteq$ $\left(\boldsymbol{F}((\Delta)), v_{t}\right)$, and therefore $\left(F((t))^{\mathbb{Q}}, v_{t}\right) \subseteq\left(\boldsymbol{F}((\Delta)), v_{t}\right)$. Since the theory of divisible ordered abelian groups is model complete (see, e.g., [Prestel and Delzell 2011, Thm. 4.1.1]),

$$
v_{t} F((t))^{\mathbb{Q}}=\mathbb{Q} \preceq \Delta=v_{t} \boldsymbol{F}((\Delta)) .
$$


Moreover,

$$
F((t))^{\mathbb{Q}} v_{t}=F \preceq \boldsymbol{F}=\boldsymbol{F}((\Delta)) v_{t} .
$$

Thus, since $\left(F((t))^{\mathbb{Q}}, v_{t}\right)$ is tame by Proposition 4.6 and $\left(\boldsymbol{F}((\Delta)), v_{t}\right)$ is tame by Lemma 4.1, Theorem 3.3 implies that

$$
\left(F((t))^{\mathbb{Q}}, v_{t}\right) \preceq\left(\boldsymbol{F}((\Delta)), v_{t}\right) .
$$

Therefore, $\left(F((t))^{\mathbb{Q}}, v_{t}\right) \models \phi$.

Let $E$ be a finite extension of $F((t))$ that contains witnesses to the truth of $\phi$ in $\left(F((t))^{\mathbb{Q}}, v_{t}\right)$. Thus $\left(E, v_{t}\right) \models \phi$. By Corollary 4.3 , there is an $\mathcal{L}_{\mathrm{vf}}(F)$-isomorphism

$$
f:\left(E, v_{t}\right) \rightarrow\left(F((t)), v_{t}\right) .
$$

Thus $\left(F((t)), v_{t}\right) \models \phi$. By Lemma 4.5,

$$
\left(F(t)^{h}, v_{t}\right) \preceq \exists\left(F((t)), v_{t}\right),
$$

hence $\left(F(t)^{h}, v_{t}\right) \models \phi$, as claimed.

Definition 5.2. Let $\boldsymbol{H}(F / C)$ be the class of tuples $(K, v, D, i)$, where $(K, v, D)$ is an $\mathcal{L}_{\mathrm{vf}}(C)$-structure and $i: F \rightarrow K v$ is a map such that

(1) $(K, v)$ is an equicharacteristic henselian nontrivially valued field,

(2) $c \mapsto d_{c}$ is an $\mathcal{L}_{\text {ring-embedding }} C \rightarrow K$,

(3) the valuation is trivial on $D$, and

(4) $i:(F, C) \rightarrow(K v, D v)$ is an $\mathcal{L}_{\text {ring }}(C)$-embedding.

Lemma 5.3 (going up from $\left.F(t)^{h}\right)$. Let $\phi$ be an $\exists-\mathcal{L}_{\mathrm{vf}}$-sentence with parameters from $C$ and the residue sort of $\left(F(t)^{h}, v_{t}\right)$, and suppose that $\left(F(t)^{h}, v_{t}, C\right) \models \phi$. Then, for all $(K, v, D, i) \in \boldsymbol{H}(F / C)$, we have that $(K, v, D) \models \phi$ (where we replace the parameters from the residue sort by their images under the map $i)$.

Proof. Write $\phi=\exists \boldsymbol{x} \psi(\boldsymbol{x} ; \boldsymbol{c}, \beta)$ for some quantifier-free formula $\psi$ and parameters $\boldsymbol{c}$ from $C$ and $\beta$ from $F(t)^{h} v_{t}$. Note that the variables in the tuple $\boldsymbol{x}$ may be from any sorts. Let $\boldsymbol{a}$ be such that

$$
\left(F(t)^{h}, v_{t}, C\right) \models \psi(\boldsymbol{a} ; \boldsymbol{c}, \beta) .
$$

Since $F(t)^{h}$ is the directed union of fields $E_{0}(t)^{h}$ for finitely generated subfields $E_{0}$ of $F$, there exists a subfield $E$ of $F$ containing $C$ such that $E / C$ is finitely generated, $\boldsymbol{a} \in E(t)^{h}$, and $\beta \in E(t)^{h} v_{t}$. Thus

$$
\left(E(t)^{h}, v_{t}, C\right) \models \psi(\boldsymbol{a} ; \boldsymbol{c}, \beta) .
$$

Since $F / C$ is separable and $E / C$ is finitely generated, $E$ is separably generated over $C$. Thus $i(E) / D v$ is separably generated. Note that the map $D v \rightarrow D$ given 
by $d_{c} v \mapsto d_{c}$ is a partial section. By Lemma 2.3 we may extend it to a partial section $g: i(E) \rightarrow K$. Let $h:=\left.g \circ i\right|_{E}$ be the composition. Then

$$
h:\left(E, v_{0}, C\right) \rightarrow(K, v, D)
$$

is an $\mathcal{L}_{\mathrm{vf}}(C)$-embedding, where $v_{0}$ denotes the trivial valuation on $E$ :

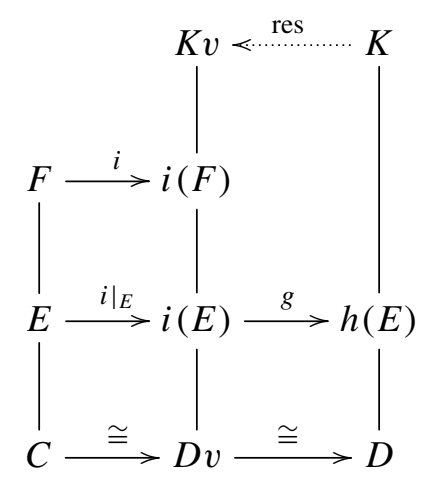

Since $(K, v)$ is nontrivial, there exists $s \in K^{\times}$with $v(s)>0$, which must be transcendental over $h(E)$, since $v$ is trivial on $h(E)$. As the rational function field $E(t)$ admits (up to equivalence) only one valuation which is trivial on $E$ and positive on $t$, we may extend $h$ to an $\mathcal{L}_{\text {vf }}(C)$-embedding

$$
h^{\prime}:\left(E(t), v_{t}, C\right) \rightarrow(K, v, D)
$$

by sending $t \mapsto s$. Since $(K, v)$ is henselian, there is a unique extension of $h^{\prime}$ to an $\mathcal{L}_{\mathrm{vf}}(C)$-embedding

$$
h^{\prime \prime}:\left(E(t)^{h}, v_{t}, C\right) \rightarrow(K, v, D) .
$$

So, since existential sentences "go up",

$$
(K, v, D) \models \psi\left(h^{\prime \prime}(\boldsymbol{a}) ; h^{\prime \prime}(\boldsymbol{c}), h^{\prime \prime}(\beta)\right),
$$

and thus $(K, v, D) \models \phi$, as claimed.

Definition 5.4. We let $R_{F / C}$ be the $\mathcal{L}_{\text {ring }}(C)$-theory of $F$ and let $R_{F / C}^{1}$ be the subtheory consisting of existential and universal sentences. Let $\boldsymbol{T}_{F / C}$ (respectively, $\boldsymbol{T}_{F / C}^{1}$ ) be the $\mathcal{L}_{\mathrm{vf}}(C)$-theory consisting of the following axioms (expressed informally about a structure $(K, v, D))$ :

(1) $(K, v)$ is an equicharacteristic henselian nontrivially valued field,

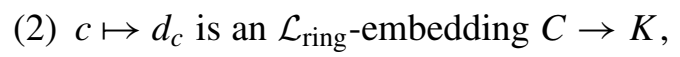

(3) the valuation $v$ is trivial on $D$, and

(4) $(K v, D v)$ is a model of $R_{F / C}$ (respectively, $R_{F / C}^{1}$ ). 
The " 1 " is intended to suggest that the sentences considered contain only one type of quantifier. Note that for any $(K, v, D) \models \boldsymbol{T}_{F / C}^{1}$, the map $d_{c} v \mapsto d_{c}$ is a partial section of the residue map. Let $\phi$ be an $\forall^{k} \exists$-sentence and write $\phi=\forall^{k} \boldsymbol{x} \psi(\boldsymbol{x})$ for some $\exists$-formula $\psi(\boldsymbol{x})$ with free variables $\boldsymbol{x}$ belonging to the residue field sort. Let ${ }^{x} K v$ denote the set of $\boldsymbol{x}$-tuples from $K v$. Then we observe that $(K, v, D) \models \phi$ if and only if ${ }^{x} K v \subseteq \psi(K)$. In this next proposition we show that, roughly, if $\boldsymbol{T}_{F / C}$ is consistent with the property " $x \subseteq \psi$ " then in fact $\boldsymbol{T}_{F \text { perf }} / C^{\text {perf }}$ entails " $x \subseteq \psi$ ".

Proposition 5.5 (main proposition). Let $\psi(x)$ be an $\exists-\mathcal{L}_{\mathrm{vf}}(C)$-formula with free variables $\boldsymbol{x}$ belonging to the residue field sort. Suppose there exists

$$
(K, v, D) \models \boldsymbol{T}_{F / C} \cup\left\{\forall^{k} \boldsymbol{x} \psi(\boldsymbol{x})\right\} .
$$

Then, for all $(L, w, E, i) \in \boldsymbol{H}\left(F^{\text {perf }} / C^{\text {perf }}\right)$, we have ${ }^{x} i(F) \subseteq \psi(L)$.

Proof. Since $(K, v, D)$ models $\boldsymbol{T}_{F / C}$, we have $(K v, D v) \equiv(F, C)$. Passing, if necessary, to an elementary extension of $(K, v, D)$, there is an elementary embedding

$$
f:(F, C) \stackrel{\preceq}{\rightarrow}(K v, D v) .
$$

As noted after the definition of $\boldsymbol{T}_{F / C}$, the map $g_{0}: D v \rightarrow D$ given by $d_{c} v \mapsto d_{c}$ is a partial section. Since $F / C$ is separable, $f(F) / D v$ is also separable. Thus any finitely generated subextension of $f(F) / D v$ is separably generated. By Lemma 2.3 we may pass again, if necessary, to an elementary extension and extend $g_{0}$ to a partial section $g: f(F) \rightarrow K$. Note that $g$ is also an $\mathcal{L}_{\text {ring }}(C)$-embedding $(f(F), D v) \rightarrow(K, D)$.

Let $h:=g \circ f$. Then $h:(F, C) \rightarrow(K, D)$ is an $\mathcal{L}_{\text {ring }}(C)$-embedding. Because $g$ is a section, the valuation $v$ is trivial when restricted to the image of $h$. Thus, if $v_{0}$ denotes the trivial valuation on $F$, the map $h$ is an $\mathcal{L}_{\mathrm{vf}}(C)$-embedding $\left(F, v_{0}, C\right) \rightarrow(K, v, D)$. The induced embedding of residue fields $\bar{h}: F v_{0} \rightarrow K v$ is the composition of the elementary embedding $f$ with an isomorphism. Thus $\bar{h}: F v_{0} \rightarrow K v$ is an elementary embedding. From now on we identify $\left(F, v_{0}, C\right)$ with its image under $h$ as a substructure of $(K, v, D)$, noting that the residue field extension is an elementary extension.

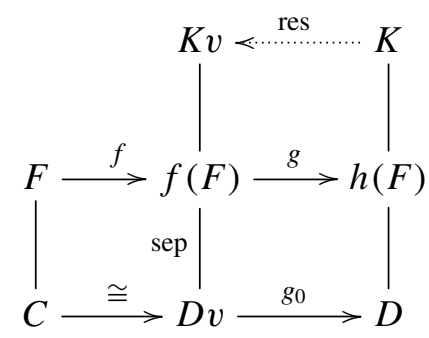


Choose an extension $\left(K^{t}, v^{t}\right) /(K, v)$ as in Proposition 2.4. Since $K^{t}$ is perfect, we can embed $D^{\text {perf }}$ into $K^{t}$ over $D$ so that $\left(K^{t}, v^{t}, D^{\text {perf }}\right)$ is an $\mathcal{L}_{\text {vf }}\left(C^{\text {perf }}\right)$ structure. Furthermore $\left(F^{\text {perf }}, v_{0}, C^{\text {perf }}\right)$ is naturally (identified with) a substructure of $\left(K^{t}, v^{t}, D^{\text {perf }}\right)$. Since $F v_{0} \preceq K v$, Lemma 3.1 gives that

$$
F^{\text {perf }} v_{0}=F v_{0}^{\text {perf }} \preceq_{\exists} K v^{\text {perf }}=K^{t} v^{t} .
$$

Thus there is an elementary extension $F^{\text {perf }} v_{0} \preceq \boldsymbol{F}$ and an embedding $\sigma: K^{t} v^{t} \rightarrow \boldsymbol{F}$ over $F^{\text {perf }} v_{0}$; see the diagram below.

Now we consider the two valued fields $\left(K^{t}, v^{t}\right)$ and $\left(\boldsymbol{F}\left(\left(v^{t} K^{t}\right)\right), v_{t}\right)$ with common subfield $\left(F^{\text {perf }}, v_{0}\right)$. Note that $K^{t}$ is tame by definition, and $\boldsymbol{F}\left(\left(v^{t} K^{t}\right)\right)$ is tame by Lemma 4.1. As a trivially valued field, $\left(F^{\text {perf }}, v_{0}\right)$ is defectless. The extension of value groups $v^{t} K^{t} / v_{0} F^{\text {perf }}$ is isomorphic to $v^{t} K^{t}$, thus it is torsion-free. The extension $K^{t} v^{t} / F^{\text {perf }} v_{0}$ is separable since $F^{\text {perf }} v_{0}$ is isomorphic to $F^{\text {perf }}$ which is perfect. Let $\left(\boldsymbol{F}\left(\left(v^{t} K^{t}\right)\right), v_{t}\right)^{*}$ be a $|K|^{+}$-saturated elementary extension of $\left(\boldsymbol{F}\left(\left(v^{t} K^{t}\right)\right), v_{t}\right)$. We have satisfied the hypotheses of Proposition 3.2, thus there exists an embedding

$$
\iota:\left(K^{t}, v^{t}\right) \rightarrow\left(\boldsymbol{F}\left(\left(v^{t} K^{t}\right)\right), v_{t}\right)^{*}
$$

over $\left(F^{\text {perf }}, v_{0}\right)$. As existential sentences "go up", we get that $\left(\boldsymbol{F}\left(\left(v^{t} K^{t}\right)\right), v_{t}\right)^{*}$, and therefore also $\left(\boldsymbol{F}\left(\left(v^{t} K^{t}\right)\right), v_{t}\right)$, models the existential $\mathcal{L}_{\mathrm{vf}}\left(F^{\text {perf }}\right)$-theory of $\left(K^{t}, v^{t}\right)$.

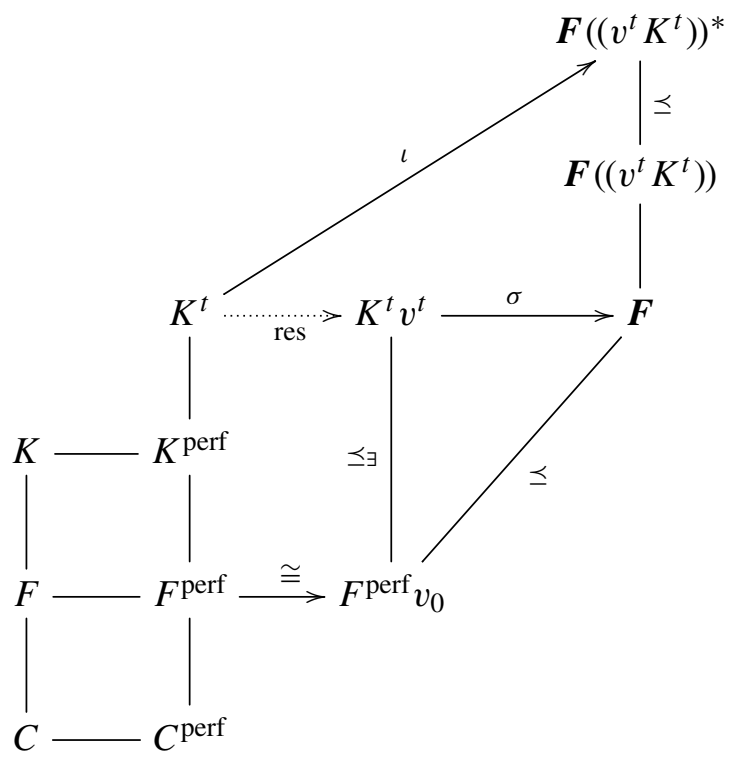

Our assumption was that $\psi(\boldsymbol{x})$ is an $\exists-\mathcal{L}_{\mathrm{vf}}(C)$-formula with free variables $\boldsymbol{x}$ belonging to the residue field sort, and that $(K, v, D) \models \forall^{k} \boldsymbol{x} \psi(\boldsymbol{x})$, i.e., ${ }^{x} K v \subseteq \psi(K)$. Then ${ }^{x} F v \subseteq{ }^{x} K v \subseteq \psi(K)$ (note that we write $F v$ rather than $F$ because we have 
identified $F$ with a subfield of $K$ ). Let

$$
\Psi_{F}:=\left\{\psi(\boldsymbol{a}) \mid \boldsymbol{a} \in{ }^{x} F v\right\} .
$$

Then $\Psi_{F}$ is a set of $\exists-\mathcal{L}_{\mathrm{vf}}(C)$-sentences (with additional parameters from $F v$ ) which is equivalent to the property that " $F v \subseteq \psi$ ". We may now restate our assumption as $(K, v) \models \Psi_{F}$. Since existential sentences "go up", $\left(K^{t}, v^{t}\right) \models \Psi_{F}$. By the result of the previous paragraph, we have $\left(\boldsymbol{F}\left(\left(v^{t} K^{t}\right)\right), v_{t}\right) \models \Psi_{F}$. By an application of Lemma 5.1, $\left(F^{\text {perf }}(t)^{h}, v_{t}\right) \models \Psi_{F}$. By Lemma 5.3, $(L, w) \models \Psi_{F}$ (where we replace the parameters from $F v$ by their images under the map $i$ ). This shows that

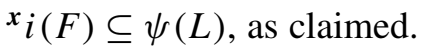

Corollary 5.6 (near $\forall^{k} \exists-C$-completeness). Let $\psi(x)$ be an $\exists-\mathcal{L}_{\mathrm{vf}}(C)$-formula with free variables $\boldsymbol{x}$ belonging to the residue field sort. Suppose there exists

$$
(K, v, D) \models \boldsymbol{T}_{F / C} \cup\left\{\forall^{k} \boldsymbol{x} \psi(\boldsymbol{x})\right\} .
$$

Then there exists $n \in \mathbb{N}$ such that ${ }^{x} L w \subseteq \psi\left(L^{p^{-n}}\right)$ for all $(L, w, E) \models \boldsymbol{T}_{F / C}$.

Proof. Let $(L, w, E) \models \boldsymbol{T}_{F / C}$. As $F / C$ is separable and $(L w, E w) \equiv(F, C)$ as $\mathcal{L}_{\text {ring }}(C)$-structures, $L w / E w$ is also separable. In particular, both $(K, v, D)$ and $(L, w, E)$ are models of $\boldsymbol{T}_{L w / E w}$, and thus we may apply the conclusion of Proposition 5.5 to

$$
\left(L^{\text {perf }}, w, E^{\text {perf }}, \mathrm{id}\right) \in \boldsymbol{H}\left(L w^{\text {perf }} / E w^{\text {perf }}\right) .
$$

Thus we have that ${ }^{x} L w \subseteq \psi\left(L^{\text {perf }}\right)$. To find $n$, we use a simple compactness argument, as follows.

Write the formula $\psi(\boldsymbol{x})$ as $\exists \boldsymbol{y} \rho(\boldsymbol{x}, \boldsymbol{y}, \boldsymbol{c})$, for a quantifier-free $\mathcal{L}_{\mathrm{vf}}$-formula $\rho$. For each $n \in \mathbb{N}$, let $\psi_{n}(\boldsymbol{x})$ be the formula $\exists \boldsymbol{y} \rho\left(\boldsymbol{x}^{p^{n}}, \boldsymbol{y}, \boldsymbol{c}^{p^{n}}\right)$ and consider the $\mathcal{L}_{\mathrm{vf}}(C)$ structure $\left(L^{p^{-n}}, w, E\right)$ which extends $(L, w, E)$. Then, for $\boldsymbol{a} \in{ }^{\boldsymbol{x}} L w, \boldsymbol{a} \in \psi\left(L^{p^{-n}}\right)$ if and only if $\boldsymbol{a} \in \psi_{n}(L)$. Let $p(\boldsymbol{x})$ be the set of formulas $\left\{\neg \psi_{n}(\boldsymbol{x}) \mid n \in \mathbb{N}\right\}$. If $p(\boldsymbol{x})$ is a type, i.e., $p(\boldsymbol{x})$ is consistent with $\boldsymbol{T}_{F / C}$, then we may realise it by a tuple $\boldsymbol{a}$ in a model $(L, w, E) \models \boldsymbol{T}_{F / C}$. Thus $\boldsymbol{a} \notin \psi\left(L^{p^{-n}}\right)$, for all $n \in \mathbb{N}$. Since $L^{\text {perf }}$ is the directed union $\bigcup_{n \in \mathbb{N}} L^{p^{-n}}$ (even as $\mathcal{L}_{\mathrm{vf}}(C)$-structures), we have that $\boldsymbol{a} \notin \psi\left(L^{\text {perf }}\right)$. This contradicts the result of the previous paragraph.

Consequently, there exists $n \in \mathbb{N}$ such that $\boldsymbol{T}_{F / C}$ entails $\forall^{k} \boldsymbol{x} \psi_{n}(\boldsymbol{x})$. Equivalently, for all $(L, w, E) \models \boldsymbol{T}_{F / C}$, we have ${ }^{x} L w \subseteq \psi\left(L^{p^{-n}}\right)$, as required.

Corollary 5.7 (perfect residue field, $\forall^{k} \exists-C$-completeness). Suppose that $F$ is perfect. Then $\boldsymbol{T}_{F / C}$ is $\forall^{k} \exists-C$-complete, i.e., for any $\forall^{k} \exists-\mathcal{L}_{\mathrm{vf}}(C)$-sentence $\phi$, either $\boldsymbol{T}_{F / C} \models \phi$ or $\boldsymbol{T}_{F / C} \models \neg \phi$.

Proof. Suppose that there is $(K, v, D) \models \boldsymbol{T}_{F / C} \cup\{\phi\}$ and let $(L, w, E) \models \boldsymbol{T}_{F / C}$. Then $(K, v, D) \models \boldsymbol{T}_{L w / E w}$ and

$$
(L, w, E, \text { id }) \in \boldsymbol{H}(L w / E w)=\boldsymbol{H}\left(L w^{\text {perf }} / E w^{\text {perf }}\right) .
$$


We write $\phi=\forall^{k} \boldsymbol{x} \psi(\boldsymbol{x})$ for some $\exists-\mathcal{L}_{\mathrm{vf}}(C)$-formula $\psi(\boldsymbol{x})$ with free variables $\boldsymbol{x}$ belonging to the residue field sort. Then $(K, v, D) \models \phi$ means that ${ }^{x} K v \subseteq \psi(K)$. Applying Proposition 5.5, we have that ${ }^{x} L w \subseteq \psi(L)$. Thus $(L, w, E) \models \phi$. This shows that $\boldsymbol{T}_{F / C} \models \phi$, as required.

Remark 5.8. We do not know whether the assumption that $F$ is perfect is necessary in Corollary 5.7. However, note that Corollary 5.7 cannot be extended from $\forall^{k} \exists$ sentences to arbitrary $\forall \exists$-sentences (even without parameters and with only one universal quantifier). For example, the sentence

$$
\forall x \exists y\left(v(x)=v\left(y^{2}\right)\right)
$$

expresses 2-divisibility of the value group, so is satisfied in $F((\mathbb{Q}))$ but not in $F((t))$.

On the other hand, one could generalise Corollary 5.7 by slightly adapting the proof to allow also sentences with more general quantifiers over the residue field, namely $Q^{k} \exists-\mathcal{L}_{\text {vf }}(C)$-sentences, i.e., sentences of the form

$$
\exists^{k} \boldsymbol{x}_{1} \forall^{k} \boldsymbol{y}_{1} \ldots \exists^{k} \boldsymbol{x}_{n} \forall^{k} \boldsymbol{y}_{n} \psi\left(\boldsymbol{x}_{1}, \boldsymbol{y}_{1}, \ldots, \boldsymbol{x}_{n}, \boldsymbol{y}_{n}\right)
$$

with $\psi\left(\boldsymbol{x}_{1}, \boldsymbol{y}_{1}, \ldots, \boldsymbol{x}_{n}, \boldsymbol{y}_{n}\right)$ an $\exists-\mathcal{L}_{\mathrm{vf}}(C)$-formula.

\section{The existential theory}

We now restrict the machinery of the previous section to existential sentences and prove Theorem 1.1 from the introduction. We fix a field $F$, let $C$ be the prime field of $F$, and write $\boldsymbol{T}_{F}=\boldsymbol{T}_{F / C}, \boldsymbol{H}(F)=\boldsymbol{H}(F / C)$.

Lemma 6.1. $\boldsymbol{T}_{F}$ is $\exists$-complete, i.e., for any $\exists-\mathcal{L}_{\mathrm{vf}}$-sentence $\phi$, either $\boldsymbol{T}_{F} \models \phi$ or $\boldsymbol{T}_{F} \models \neg \phi$.

Proof. Suppose that $\boldsymbol{T}_{F} \cup\{\phi\}$ is consistent. Thus there exists $(K, v) \models \boldsymbol{T}_{F} \cup\{\phi\}$. Simply viewing $\phi$ as an $\forall^{k} \exists$-formula $\forall^{k} x \psi(x)$ with $\psi(x)=\phi$, we have that $K v \subseteq \psi(K)$. By Corollary 5.6 there exists $n \in \mathbb{N}$ such that, for every $(L, w) \models \boldsymbol{T}_{F}$, $L w \subseteq \psi\left(L^{p^{-n}}\right)$. In particular, $\psi\left(L^{p^{-n}}\right)$ is nonempty. Since no parameters appear in $\psi$, we may apply the $n$-th power of the Frobenius map to get that $\psi(L)$ is nonempty, for every $(L, w) \models \boldsymbol{T}_{F}$. Viewing $\phi$ as an $\exists$-sentence again, we have that $(L, w) \models \phi$. Thus $\boldsymbol{T}_{F} \models \phi$, as required.

For the proof of Theorem 1.1 it remains to show that $\boldsymbol{T}_{F}^{1}$ already entails those existential and universal sentences which are entailed by $\boldsymbol{T}_{F}$.

Definition 6.2. We define two subtheories of $\boldsymbol{T}_{F}^{1}$. Let $T_{F}^{\exists}$ be the $\mathcal{L}_{\mathrm{vf}}$-theory consisting of the following axioms (expressed informally about a structure $(K, v)$ ):

(1) $(K, v)$ is an equicharacteristic henselian nontrivially valued field and

(2) $K v$ is a model of the existential $\mathcal{L}_{\text {ring }}$-theory of $F$. 
Let $T_{F}^{\forall}$ be the $\mathcal{L}_{\mathrm{vf}}$-theory consisting of the following axioms (again expressed informally):

(1) $(K, v)$ is an equicharacteristic henselian nontrivially valued field and

(2) $K v$ is a model of the universal $\mathcal{L}_{\text {ring }}$-theory of $F$.

Note that $\boldsymbol{T}_{F}^{1} \equiv T_{F}^{\exists} \cup T_{F}^{\forall}$.

Lemma 6.3. Let $\phi$ be an existential $\mathcal{L}_{\mathrm{vf}}$-sentence. If $\boldsymbol{T}_{F} \models \phi$ then $T_{F}^{\exists} \models \phi$.

Proof. Let $(K, v) \models T_{F}^{\exists}$. Then $K v$ is a model of $\operatorname{Th}_{\exists}(F)$; equivalently the theory of $K v$ is consistent with the atomic diagram of $F$. Thus there is an elementary extension $(K, v) \preceq\left(K^{*}, v^{*}\right)$ with an embedding $\sigma: F \rightarrow K^{*} v^{*}$, cf. [Marker 2002, Lemma 2.3.3]. Note that $\left(K^{*}, v^{*}, \sigma\right) \in \boldsymbol{H}(F)$ and that $\left(F(t)^{h}, v_{t}\right) \models \boldsymbol{T}_{F}$, hence $\left(F(t)^{h}, v_{t}\right) \models \phi$. Therefore, Lemma 5.3 implies that $\left(K^{*}, v^{*}\right) \models \phi$; thus $(K, v) \models \phi$. This shows that $T_{F}^{\exists}=\phi$.

Lemma 6.4. Let $\phi$ be a universal $\mathcal{L}_{\mathrm{vf}}$-sentence. If $\boldsymbol{T}_{F} \models \phi$ then $T_{F}^{\forall} \models \phi$.

Proof. Let $(K, v) \models T_{F}^{\forall}$. Then $K v \models \mathrm{Th}_{\forall}(F)$. There exists $F^{\prime} \equiv F$ with an embedding $\sigma: K v \rightarrow F^{\prime}$ (see [Marker 2002, Exercise 2.5.10]). Using Lemma 2.1, we may choose an equicharacteristic nontrivially valued field $(L, w)$ which extends $(K, v)$ and is such that $L w$ is isomorphic to $F^{\prime}$. In particular $L w \equiv F$. Let $(L, w)^{h}$ be the henselisation of $(L, w)$; then we have $(L, w)^{h} \models \boldsymbol{T}_{F}$, so $(L, w)^{h} \models \phi$. Since $\phi$ is universal, we conclude that $(K, v) \models \phi$.

Theorem 6.5 ( $\exists$-completeness). $\boldsymbol{T}_{F}^{1}$ is $\exists$-complete, i.e., for any $\exists-\mathcal{L}_{\mathrm{vf}}$-sentence $\phi$ either $\boldsymbol{T}_{F}^{1} \models \phi$ or $\boldsymbol{T}_{F}^{1} \models \neg \phi$.

Proof. Let $\phi$ be an existential $\mathcal{L}_{\text {vf }}$-sentence. By Lemma 6.1, either $\boldsymbol{T}_{F} \models \phi$ or $\boldsymbol{T}_{F} \models \neg \phi$. In the first case we apply Lemma 6.3 and find that $T_{F}^{\exists} \models \phi$; in the second case we apply Lemma 6.4 and find that $T_{F}^{\forall} \models \neg \phi$. Since $\boldsymbol{T}_{F}^{1} \equiv T_{F}^{\exists} \cup T_{F}^{\forall}$, in either case $\boldsymbol{T}_{F}^{1}$ "decides" $\phi$, and we are done.

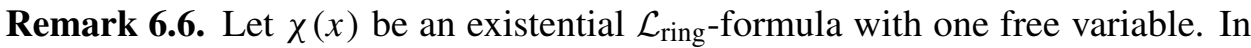
[Anscombe and Fehm 2016], we apply Theorem 6.5 to the following $\exists-$ or $\forall-\mathcal{L}_{\mathrm{vf}^{-}}$ sentences:

(1) $\forall x(\chi(x) \rightarrow v(x) \geq 0)$,

(2) $\forall x(\chi(x) \rightarrow v(x)>0)$, and

(3) $\exists x(v(x)>0 \wedge x \neq 0 \wedge \chi(x))$.

We also apply Corollary 5.6 to the $\forall^{k} \exists-\mathcal{L}_{\mathrm{vf}}$-sentence

(4) $\forall^{k} x \exists y(\operatorname{res}(y)=x \wedge \chi(y))$. 


\section{An "existential AKE principle" and existential decidability}

Theorem 6.5 shows that the existential (respectively, universal) theory of an equicharacteristic henselian nontrivially valued field depends only on the existential (respectively, universal) theory of its residue field. We formulate this in the following "existential AKE principle".

Theorem 7.1. Let $(K, v)$ and $(L, w)$ be equicharacteristic henselian nontrivially valued fields. Then

$$
(K, v) \models \mathrm{Th}_{\exists}(L, w) \quad \text { if and only if } \quad K v \models \mathrm{Th}_{\exists}(L w) .
$$

Proof. $(\Rightarrow)$ : Note that the maximal ideal is defined by the quantifier-free formula $v(x)>0$. Therefore any existential statement about the residue field can be translated into an existential statement about the valued field.

$(\Leftarrow)$ : If $K v \models \mathrm{Th}_{\exists}(L w)$ then $(K, v) \models T_{L w}^{\exists}$. By Lemma 6.1, $\boldsymbol{T}_{L w}$ entails the existential theory of $(L, w)$, and by Lemma $6.3, T_{L w}^{\exists}$ entails the existential consequences of $\boldsymbol{T}_{L w}$. Combining these two statements, we have that $T_{L w}^{\exists}$ entails the existential theory of $(L, w)$. Thus $(K, v)$ models the existential theory of $(L, w)$.

Corollary 7.2. Let $(K, v)$ and $(L, w)$ be equicharacteristic henselian nontrivially valued fields. Then

$$
\mathrm{Th}_{\exists}(K, v)=\mathrm{Th}_{\exists}(L, w) \quad \text { if and only if } \quad \mathrm{Th}_{\exists}(K v)=\mathrm{Th}_{\exists}(L w) .
$$

Proof. This follows from Theorem 7.1, since $\operatorname{Th}_{\exists}(K, v)=\mathrm{Th}_{\exists}(L, w)$ if and only if both $(K, v) \models \operatorname{Th}_{\exists}(L, w)$ and $(L, w) \models \operatorname{Th}_{\exists}(K, v)$, and $\operatorname{Th}_{\exists}(K v)=\operatorname{Th}_{\exists}(L w)$ if and only if both $K v \models \mathrm{Th}_{\exists}(L w)$ and $L w \models \mathrm{Th}_{\exists}(K v)$.

Note that Corollary 7.2 is in fact simply a reformulation of Theorem 6.5. Note moreover that, by the usual duality between existential and universal sentences, the same principle holds with " $\exists$ " replaced by " $\forall$ ".

Remark 7.3. The reader has probably noticed that as opposed to the usual AKE principles, the value group does not occur here. However, since the existential theory of a valued field determines the existential theory of its value group, Corollary 7.2 could also be phrased as

$\operatorname{Th}_{\exists}(K, v)=\operatorname{Th}_{\exists}(L, w) \quad$ if and only if

$$
\mathrm{Th}_{\exists}(K v)=\mathrm{Th}_{\exists}(L w) \text { and } \mathrm{Th}_{\exists}(v K)=\mathrm{Th}_{\exists}(w L) .
$$

In fact, all nontrivial ordered abelian groups have the same existential theory (which follows immediately from the completeness of the theory of divisible ordered abelian groups; see also [Gurevich and Kokorin 1963]). In residue characteristic zero, this special form of the existential AKE principle was known before; see, e.g., [Koenigsmann 2014, p. 192]. 
Remark 7.4. In mixed characteristic the situation is very different. Fix a prime $p$ and let $(K, v)$ and $(L, w)$ be henselian nontrivially valued fields. Just as in Remark 7.3, the existential theory of a valued field determines the existential theory of the residue field and the value group, i.e.,

$\mathrm{Th}_{\exists}(K, v)=\mathrm{Th}_{\exists}(L, w) \quad \Longrightarrow \quad \mathrm{Th}_{\exists}(K v)=\mathrm{Th}_{\exists}(L w)$ and $\mathrm{Th}_{\exists}(v K)=\mathrm{Th}_{\exists}(w L)$.

However, in mixed characteristic the converse fails. For example, consider the valued fields $(K, v)=\left(\mathbb{Q}_{p}, v_{p}\right)$ and $(L, w)=\left(\mathbb{Q}_{p}(\sqrt{p}), v_{p}\right)$. Both residue fields $K v$ and $L w$ are equal to $\mathbb{F}_{p}$ and both value groups are isomorphic to $\mathbb{Z}$, but the existential theories of $(K, v)$ and $(L, w)$ are not equal since $\mathbb{Q}_{p}$ does not contain a square-root of $p$. In particular, both Theorem 6.5 and Corollary 7.2 fail if we replace "equicharacteristic" by "mixed characteristic".

One feature of mixed characteristic is that the existential theory of $(K, v)$ determines the existential theory of $(v K, v p)$, which is the ordered abelian group $v K$ together with the distinguished nonzero element $v p$. Therefore, if $(K, v)$ and $(L, w)$ are both of characteristic zero and residue characteristic $p$, we have the implication

$$
\begin{aligned}
\operatorname{Th}_{\exists}(K, v)=\operatorname{Th}_{\exists}(L, w) & \Rightarrow \\
\operatorname{Th}_{\exists}(K v) & =\operatorname{Th}_{\exists}(L w) \text { and } \operatorname{Th}_{\exists}(v K, v p)=\operatorname{Th}_{\exists}(w L, w p) .
\end{aligned}
$$

Note that not all ordered abelian groups with a distinguished nonzero element have the same existential theory. For example, $\mathrm{Th}_{\exists}(\mathbb{Z}, 1) \neq \mathrm{Th}_{\exists}(\mathbb{Z}, 2)$. Nevertheless, we claim that the implication $(*)$ is not invertible. To prove this claim we need a new counterexample because $v_{p} p$ is minimal positive in $v_{p} \mathbb{Q}_{p}$ but $v_{p} p=2 v_{p} \sqrt{p}$ in $v_{p} \mathbb{Q}_{p}(\sqrt{p})$, and so

$$
\mathrm{Th}_{\exists}\left(v_{p} \mathbb{Q}_{p}, v_{p} p\right)=\mathrm{Th}_{\exists}(\mathbb{Z}, 1) \neq \operatorname{Th}_{\exists}(\mathbb{Z}, 2)=\operatorname{Th}_{\exists}\left(v_{p} \mathbb{Q}_{p}(\sqrt{p}), v_{p} p\right) .
$$

Instead, we cite the example of two valued fields $\left(L_{1}, v\right)$ and $\left(F_{1}, v\right)$ which were constructed in [Anscombe and Kuhlmann 2016, Theorem 1.5]. Both are tame and algebraic extensions of $\left(\mathbb{Q}, v_{p}\right)$, both residue fields $L_{1} v$ and $F_{1} v$ are equal to $\mathbb{F}_{p}$, and both value groups $v L_{1}$ and $v F_{1}$ are equal to the $p$-divisible hull of $\frac{1}{p-1}\left(v_{p} p\right) \mathbb{Z}$. Nevertheless $\left(L_{1}, v\right) \not \equiv\left(F_{1}, v\right)$. In fact, since $L_{1}$ and $F_{1}$ are algebraic, we have that $\mathrm{Th}_{\exists}\left(L_{1}, v\right) \neq \mathrm{Th}_{\exists}\left(F_{1}, v\right)$. This example shows that the converse to $(*)$ does not hold, even under the additional hypothesis that $(K, v)$ and $(L, w)$ are tame.

Next we deduce Corollary 1.3 from Theorem 6.5.

Corollary 7.5. Let $(K, v)$ be an equicharacteristic henselian valued field. The following are equivalent.

(1) $\mathrm{Th}_{\exists}(K v)$ is decidable.

(2) $\mathrm{Th}_{\exists}(K, v)$ is decidable. 
Proof. $2 \Longrightarrow 1$ : As before, residue fields are interpreted in valued fields in such a way that existential statements about $K v$ remain existential statements about $(K, v)$. Therefore, if $(K, v)$ is $\exists$-decidable, then $K v$ is $\exists$-decidable.

$1 \Longrightarrow 2$ : Write $F:=K v$ and suppose that $F$ is $\exists$-decidable. If $v$ is trivial, then $(K, v)=(F, v)$ is also $\exists$-decidable, so suppose that $v$ is nontrivial. We may recursively enumerate the existential and universal theory $R_{F}^{1}$ of $F$, so $T_{F}^{1}$ is effectively axiomatisable. By Theorem $6.5, \boldsymbol{T}_{F}^{1}$ is an $\exists$-complete subtheory of $\operatorname{Th}(K, v)$. Thus we may decide the truth of existential (and universal) sentences in $(K, v)$.

Remark 7.6. If we replace "equicharacteristic" by "mixed characteristic" then the statement of Corollary 7.5 is no longer true. To see this, let $P$ be an undecidable set of primes, let $K$ be the extension of $\mathbb{Q}_{p}$ generated by a family of $l$-th roots of $p$, for

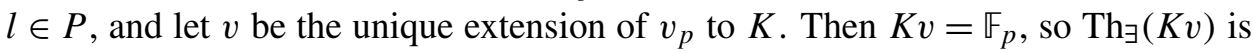
decidable, but $\operatorname{Th}_{\exists}(v K, v p)$ is undecidable, hence so is $\operatorname{Th}_{\exists}(K, v)$. At present, we do not know of an example of a mixed characteristic henselian valued field $(K, v)$ for which $\mathrm{Th}_{\exists}(K v)$ and $\mathrm{Th}_{\exists}(v K, v p)$ are decidable but $\mathrm{Th}_{\exists}(K, v)$ is undecidable.

Let $\mathcal{L}_{\mathrm{vf}}(t)$ be the language of valued fields with an additional parameter $t$, and let $q$ be a prime power. In [Denef and Schoutens 2003], it is shown that resolution of singularities in characteristic $p$ would imply that the existential $\mathcal{L}_{\mathrm{vf}}(t)$-theory of $\mathbb{F}_{q}((t))$ is decidable. Using our methods we can prove the following weaker but unconditional result.

Corollary 7.7. The existential theory of $\mathbb{F}_{q}((t))$ in the language of valued fields is decidable.

First proof. We can apply Corollary 7.5, noting that $\mathrm{Th}_{\exists}\left(\mathbb{F}_{q}\right)$ is decidable.

For the sake of interest, we present a more direct proof of this special case. However, note that this "second proof" uses the decidability of $\mathbb{F}_{q}$, while the "first proof" used only the decidability of the existential theory of $\mathbb{F}_{q}$.

Second proof. As an equicharacteristic tame field (Proposition 4.6) with decidable residue field and value group, $\left(\mathbb{F}_{q}((t))^{\mathbb{Q}}, v_{t}\right)$ is decidable, by [Kuhlmann 2014, Theorem 7.7(a)]. Since $\left(\mathbb{F}_{q}((t))^{\mathbb{Q}}, v_{t}\right)$ is the directed union of structures isomorphic to $\left(\mathbb{F}_{q}((t)), v_{t}\right)$ (Corollary 4.3), in fact $\left(\mathbb{F}_{q}((t)), v_{t}\right)$ and $\left(\mathbb{F}_{q}((t))^{\mathbb{Q}}, v_{t}\right)$ have the same $\exists$ - $\mathcal{L}_{\text {vf }}$-theory. Thus, to decide the existential $\mathcal{L}_{\mathrm{vf}}$-theory of $\left(\mathbb{F}_{q}((t)), v_{t}\right)$, it suffices to apply the decision procedure for the $\mathcal{L}_{\mathrm{vf}}$-theory of $\left(\mathbb{F}_{q}((t))^{\mathbb{Q}}, v_{t}\right)$.

Remark 7.8. Since Corollary 7.7 shows decidability of the existential theory of $\mathbb{F}_{q}((t))$ in the language of valued fields $\mathcal{L}_{\mathrm{vf}}$, in which the valuation ring is definable by a quantifier-free formula, we also get decidability of the existential theory of the ring $\mathbb{F}_{q} \llbracket t \rrbracket$. It might however be interesting to point out that it was proven only recently that already decidability of the existential theory of $\mathbb{F}_{q}((t))$ in the language 
of rings would imply decidability of the existential theory of the ring $\mathbb{F}_{q} \llbracket t \rrbracket$; see [Anscombe and Koenigsmann 2014, Corollary 3.4].

Remark 7.9. The $\exists-\mathcal{L}_{\mathrm{vf}}(t)$-theory of $\left(\mathbb{F}_{q}((t)), v_{t}\right)$ is equivalent to the $\forall_{1}^{K} \exists-\mathcal{L}_{\mathrm{vf}}{ }^{-}$ theory of $\left(\mathbb{F}_{q}((t)), v_{t}\right)$. This "equivalence" is meant in the sense that there is a truthpreserving effective translation between $\exists-\mathcal{L}_{\mathrm{vf}}(t)$-sentences and $\forall \exists-\mathcal{L}_{\mathrm{vf}}$-sentences which have only one universal quantifier ranging over the valued field sort (and arbitrary existential quantifiers). In this argument we make repeated use of the fact that, for all $a \in \mathbb{F}_{q}((t))$ with $v_{t}(a)>0$ and $a \neq 0$, there is an $\mathcal{L}_{\mathrm{vf}}$-embedding $\mathbb{F}_{q}((t)) \rightarrow \mathbb{F}_{q}((t))$ which sends $t \mapsto a$.

Let $\phi(t)$ be an existential $\mathcal{L}_{\mathrm{vf}}(t)$-sentence. We claim that $\phi(t)$ is equivalent to the $\forall_{1}^{K} \exists-\mathcal{L}_{\mathrm{vf}}$-sentence

$$
\forall u((v(u)>0 \wedge u \neq 0) \rightarrow \phi(u)) .
$$

This follows from the fact about embeddings stated above.

On the other hand, let $\psi(x)$ be an $\exists-\mathcal{L}_{\mathrm{vf}}$-formula in one free variable $x$ in the valued field sort and consider the $\exists-\mathcal{L}_{\mathrm{vf}}(t)$-sentence $\chi$ which is defined to be $\exists y \exists z_{0} \ldots \exists z_{q-1}\left(y t=1 \wedge \psi(y) \wedge \bigwedge_{j} z_{j}^{q}=z_{j} \wedge \bigwedge_{i \neq j} z_{i} \neq z_{j} \wedge \bigwedge_{j} \psi\left(z_{j}+t\right) \wedge \bigwedge_{j} \psi\left(z_{j}\right)\right)$.

Written more informally, the sentence $\chi$ expresses that

$$
\psi\left(t^{-1}\right) \wedge \bigwedge_{z \in \mathbb{F}_{q}}(\psi(z+t) \wedge \psi(z))
$$

We claim that $\forall x \psi(x)$ and $\chi$ are equivalent. First suppose that $\mathbb{F}_{q}((t)) \models \forall x \psi(x)$. By choosing $\left(z_{j}\right)$ to be an enumeration of $\mathbb{F}_{q}$, we immediately have that $\mathbb{F}_{q}((t)) \models \chi$.

In the other direction, suppose that $\mathbb{F}_{q}((t)) \models \chi$ and let $a \in \mathbb{F}_{q}((t))$. If $v_{t}(a)<0$ then consider the embedding which sends $t \mapsto a^{-1}$. Since $\psi\left(t^{-1}\right)$ holds, applying the embedding shows that $\psi(a)$ also holds. On the other hand suppose that $v_{t}(a) \geq 0$. If $a \in \mathbb{F}_{q}$ then $\chi$ already entails that $\psi(a)$. Now suppose that $a \notin \mathbb{F}_{q}$ and let $z$ be the residue of $a$. Consider the embedding which sends $t \mapsto a-z$ (note that $a-z \neq 0$ ). Since $\psi(z+t)$ holds, applying the embedding shows that $\psi(a)$ also holds. This completes the proof that $\mathbb{F}_{q}((t)) \models \forall x \psi(x)$.

\section{Acknowledgements}

The authors would like to thank Immanuel Halupczok, Ehud Hrushovski, Jochen Koenigsmann, Dugald Macpherson, and Alexander Prestel for helpful discussions and encouragement.

\section{References}

[Anscombe and Fehm 2016] S. Anscombe and A. Fehm, "Characterizing diophantine henselian valuation rings and valuation ideals", preprint, 2016. arXiv 
[Anscombe and Koenigsmann 2014] W. Anscombe and J. Koenigsmann, "An existential $\varnothing$-definition of $\mathbb{F}_{q} \llbracket t \rrbracket$ in $\mathbb{F}_{q}((t))$ ”, J. Symb. Log. 79:4 (2014), 1336-1343. MR Zbl

[Anscombe and Kuhlmann 2016] S. Anscombe and F.-V. Kuhlmann, "Notes on extremal and tame valued fields", 2016, http://math.usask.ca/ fvk/EXTRNOT.pdf. To appear in J. Sym. Logic.

[Cluckers et al. 2013] R. Cluckers, J. Derakhshan, E. Leenknegt, and A. Macintyre, "Uniformly defining valuation rings in Henselian valued fields with finite or pseudo-finite residue fields", Ann. Pure Appl. Logic 164:12 (2013), 1236-1246. MR Zbl

[Denef and Schoutens 2003] J. Denef and H. Schoutens, "On the decidability of the existential theory of $\mathbb{F}_{p} \llbracket t \rrbracket ”$, pp. 43-60 in Valuation theory and its applications (Saskatoon, 1999), vol. II, edited by F.-V. Kuhlmann et al., Fields Inst. Commun. 33, Amer. Math. Soc., Providence, RI, 2003. MR Zbl

[Efrat 2006] I. Efrat, Valuations, orderings, and Milnor K-theory, Mathematical Surveys and Monographs 124, Amer. Math. Soc., Providence, RI, 2006. MR Zbl

[Engler and Prestel 2005] A. J. Engler and A. Prestel, Valued fields, Springer, Berlin, 2005. MR Zbl

[Fehm 2015] A. Fehm, "Existential $\varnothing$-definability of Henselian valuation rings", J. Symb. Log. 80:1 (2015), 301-307. MR Zbl

[Gurevich and Kokorin 1963] Y. S. Gurevich and A. I. Kokorin, "Universal equivalence of ordered Abelian groups", Algebra i Logika Sem. 2:1 (1963), 37-39. In Russian. MR

[Koenigsmann 2014] J. Koenigsmann, "Undecidability in number theory", pp. 159-195 in Model theory in algebra, analysis and arithmetic, edited by H. D. Macpherson and C. Toffalori, Lecture Notes in Math. 2111, Springer, Heidelberg, 2014. MR Zbl

[Kuhlmann 2001] F.-V. Kuhlmann, "Elementary properties of power series fields over finite fields", $J$. Symbolic Logic 66:2 (2001), 771-791. MR Zbl

[Kuhlmann 2004] F.-V. Kuhlmann, "Value groups, residue fields, and bad places of rational function fields", Trans. Amer. Math. Soc. 356:11 (2004), 4559-4600. MR Zbl

[Kuhlmann 2011] F.-V. Kuhlmann, "Valuation theory", book in progress, 2011, http://math.usask.ca/ $\sim$ fvk/Fvkbook.htm.

[Kuhlmann 2014] F.-V. Kuhlmann, "The algebra and model theory of tame valued fields", J. Reine Angew. Math. (online publication May 2014).

[Kuhlmann et al. 1986] F.-V. Kuhlmann, M. Pank, and P. Roquette, "Immediate and purely wild extensions of valued fields", Manuscripta Math. 55:1 (1986), 39-67. MR Zbl

[Marker 2002] D. Marker, Model theory: an introduction, Graduate Texts in Mathematics 217, Springer, New York, 2002. MR Zbl

[Prestel 2015] A. Prestel, "Definable Henselian valuation rings", J. Symb. Log. 80:4 (2015), 12601267. MR Zbl

[Prestel and Delzell 2011] A. Prestel and C. N. Delzell, Mathematical logic and model theory: a brief introduction, Springer, London, 2011. MR Zbl

[Serre 1979] J.-P. Serre, Local fields, Graduate Texts in Mathematics 67, Springer, New York, 1979. MR Zbl

Communicated by Jonathan Pila

Received 2015-09-18 Revised 2016-02-09 Accepted 2016-03-15

sanscombe@uclan.ac.uk Jeremiah Horrocks Institute, University of Central Lancashire, Preston, PR1 2HE, United Kingdom

arno.fehm@manchester.ac.uk School of Mathematics, University of Manchester, Oxford Road, Manchester, M13 9PL, United Kingdom 


\section{Algebra \& Number Theory}

msp.org/ant

\section{EDITORS}

MANAGING EDITOR

Bjorn Poonen

Massachusetts Institute of Technology

Cambridge, USA

\author{
EDITORIAL BOARD CHAIR \\ David Eisenbud \\ University of California \\ Berkeley, USA
}

\section{BOARD OF EDITORS}

Georgia Benkart

Dave Benson

Richard E. Borcherds

John H. Coates

J-L. Colliot-Thélène

Brian D. Conrad

Hélène Esnault

Hubert Flenner

Sergey Fomin

Edward Frenkel

Andrew Granville

Joseph Gubeladze

Roger Heath-Brown

Craig Huneke

Kiran S. Kedlaya

János Kollár

Yuri Manin

Philippe Michel
University of Wisconsin, Madison, USA

University of Aberdeen, Scotland

University of California, Berkeley, USA

University of Cambridge, UK

CNRS, Université Paris-Sud, France

Stanford University, USA

Freie Universität Berlin, Germany

Ruhr-Universität, Germany

University of Michigan, USA

University of California, Berkeley, USA

Université de Montréal, Canada

San Francisco State University, USA

Oxford University, UK

University of Virginia, USA

Univ. of California, San Diego, USA

Princeton University, USA

Northwestern University, USA

École Polytechnique Fédérale de Lausanne
Susan Montgomery

Shigefumi Mori

Raman Parimala

Jonathan Pila

Anand Pillay

Victor Reiner

Peter Sarnak

Joseph H. Silverman

Michael Singer

Vasudevan Srinivas

J. Toby Stafford

Ravi Vakil

Michel van den Bergh

Marie-France Vignéras

Kei-Ichi Watanabe

Efim Zelmanov

Shou-Wu Zhang
University of Southern California, USA

RIMS, Kyoto University, Japan

Emory University, USA

University of Oxford, UK

University of Notre Dame, USA

University of Minnesota, USA

Princeton University, USA

Brown University, USA

North Carolina State University, USA

Tata Inst. of Fund. Research, India

University of Michigan, USA

Stanford University, USA

Hasselt University, Belgium

Université Paris VII, France

Nihon University, Japan

University of California, San Diego, USA

Princeton University, USA

\section{PRODUCTION}

production@msp.org

Silvio Levy, Scientific Editor

See inside back cover or msp.org/ant for submission instructions.

The subscription price for 2016 is US \$/year for the electronic version, and \$/year (+\$, if shipping outside the US) for print and electronic. Subscriptions, requests for back issues and changes of subscribers address should be sent to MSP.

Algebra \& Number Theory (ISSN 1944-7833 electronic, 1937-0652 printed) at Mathematical Sciences Publishers, 798 Evans Hall \#3840, c/o University of California, Berkeley, CA 94720-3840 is published continuously online. Periodical rate postage paid at Berkeley, CA 94704, and additional mailing offices.

ANT peer review and production are managed by EditFLOW ${ }^{\circledR}$ from MSP.

\section{PUBLISHED BY}

mathematical sciences publishers

nonprofit scientific publishing

http://msp.org/

(C) 2016 Mathematical Sciences Publishers 


\section{Algebra \& Number Theory}

Volume $10 \quad$ No. $3 \quad 2016$

Group schemes and local densities of ramified hermitian lattices in residue characteristic 2: Part I

SUNGMUN CHO

Presentation of affine Kac-Moody groups over rings

DANIEL ALLCOCK

Discriminant formulas and applications

Kenneth Chan, Alexander A. Young and James J. Zhang

LUIS E. GARCIA

Multiple period integrals and cohomology

RoElof W. BRUGGEMAN and YoungJu CHOIE

The existential theory of equicharacteristic henselian valued fields

SYLVY ANSCOMBE and ARNO FEHM

SOMNATH JHA, TADASHI OCHIAI and GERGELY ZÁBRÁDI 